\title{
Resistance to Fludioxonil in Botrytis cinerea Isolates from Blackberry and Strawberry
}

\author{
Xingpeng Li, Dolores Fernández-Ortuño, Anja Grabke, and Guido Schnabel
}

First, third, and fourth authors: School of Agricultural, Forest, \& Environmental Sciences, Clemson University, Clemson SC 29634; and second author: Instituto de Hortofruticultura Subtropical y Mediterránea "La Mayora"-Universidad de Málaga-Consejo Superior de Investigaciones Científica (IHSM-UMA-CSIC), Departamento de Microbiología, Campus de Teatinos, 29071 Málaga, Spain.

Accepted for publication 6 January 2014.

\begin{abstract}
Li, X., Fernández-Ortuño, D., Grabke, A., and Schnabel, G. 2014. Resistance to fludioxonil in Botrytis cinerea isolates from blackberry and strawberry. Phytopathology 104:724-732.

Site-specific fungicides, including the phenylpyrrole fludioxonil, are frequently used for gray mold control but are at risk for the development of resistance. In this study, field isolates that were low-resistant (LR) and moderately resistant (MR) to fludioxonil from blackberry and strawberry fields of North Carolina, South Carolina, and Virginia were characterized. Genes involved in osmoregulation, including bcsak1, BcOS4, bos5, and $B R R G-1$, were cloned and sequenced to detect potential target gene alterations; however, none were found. A previously described mutation (R632I) in transcription factor Mrr1, which is known to increase the expression of ATP-binding cassette transporter AtrB, was found in MR

was $\approx 200$-fold increased compared with an $\mathrm{S}$ isolate; however, 30- to 100 -fold overexpression was also detected in LR isolates. Both MR isolates exhibited increased sensitivity to salt stress in the form of mycelial growth inhibition at $4 \% \mathrm{NaCl}$, indicating a disruption of osmoregulatory processes in those strains. However, the glycerol content was indistinguishable between S, LR, and MR isolates with and without exposure to fludioxonil, suggesting that the glycerol synthesis pathway may not be a part of the resistance mechanism in LR or MR strains. An investigation into the origin of LR and MR isolates from blackberry revealed two insertions in the mrrl gene consistent with those found in the Botrytis clade group $\mathrm{S}$. The emergence of strains overexpressing atrB in European and now in North American strawberry fields underscores the importance of this resistance mechanism for development of resistance to fludioxonil in Botrytis cinerea.
\end{abstract} but not in sensitive (S) or LR isolates. Expression of atrB in MR isolates
Fludioxonil is a phenylpyrrole fungicide with broad-spectrum activity against fungal plant pathogens among ascomycetes and basidiomycetes (12). Fludioxonil is effective against most diseases of seed, including seedling blight, stem-base browning, snow mold, and common bunt. It is very effective against Botrytis cinerea Pers., the causal agent of gray mold disease of many vegetables and small fruits, and inhibits both mycelial growth and spore germination (24). It is registered in the United States against various post-harvest rots of pome fruits, stone fruits, and sweet potato. Fludioxonil was first registered in 2002 and is listed under Fungicide Resistance Action Committee (FRAC) code 12 with FRAC resistance risk category low to medium.

Resistance in $B$. cinerea to fludioxonil is rarely found in the field $(7,25,34)$ worldwide. One isolate from apple grown in Washington State was moderately resistant (MR) to fludioxonil but was impaired in fitness and pathogenicity on apple fruit (43). Isolates with reduced sensitivity to fludioxonil were found in strawberry fields in Virginia (6), Maryland, and South Carolina (8). Low-resistant (LR) and MR B. cinerea isolates were reported from European small fruit, including strawberry $(20,23)$ and grapevines $(20,22,28)$. The effective dose that inhibits $50 \%$ of mycelial growth $\left(\mathrm{EC}_{50}\right)$ values for isolates $\mathrm{MR}$ to fludioxonil from Germany and Washington were 0.04 to $1.5 \mu \mathrm{g} / \mathrm{ml}$, depending on the evaluation method $(23,43)$. High levels of resistance to fludioxonil have not been reported in the field, probably due to a fitness penalty in such isolates $(4,25)$.

Corresponding author: G. Schnabel; E-mail address: schnabe@clemson.edu

http://dx.doi.org/10.1094/PHYTO-11-13-0308-R

(C) 2014 The American Phytopathological Society
The precise mode of action of fludioxonil is still unknown but fludioxonil treatment induced cell death in filamentous fungi by improperly activating the Hog1-type mitogen-activated protein kinase (MAPK) $(19,41,42)$, indicating possible disruption of the osmoregulation pathway. High levels of fludioxonil resistance in laboratory mutants are linked to enzymes involved in osmoregulation pathway, such as histidine-kinases (HKs); specifically, the group III HK is considered to be a target for fludioxonil in yeast and filamentous fungi $(2,14,30,41)$. Some evidence was provided for fludioxonil resistance to be linked to at least two different genes (34). Genes assumed to be involved in osmoregulation in $B$. cinerea were identified by gene disruption and include bosl (9), bcsakl (32), BcOS4 (40), bos5 (39), and BRRG-1 (38). Corresponding knock-out mutants revealed reduced salt tolerance and increased resistance to fludioxonil. Hypersensitivity to osmotic stress has also been associated with lack of glycerol accumulation and resistance to the fludioxonil (18).

Moderate resistance to fludioxonil in $B$. cinerea is associated with the multidrug resistance (MDR) phenotypes MDR1 and MDR1h (20,23). Both phenotypes are associated with overexpression of the ATP-binding cassette (ABC) transporter gene atr $B$ and mutations in transcription factor Mrr1 (20,33). Although MDR1 phenotypes express atr $B \approx 50$-fold, MDR1h phenotypes express $a t r B$ at even higher levels (23). The overexpression of two other multidrug transporters has been linked to multifungicide resistance in $B$ cinerea, including $m f s M 2$ and atrD. MfsM2 belongs to the major facilitator superfamily (MFS) and overexpression is associated with increased resistance to multiple fungicides (designated MDR2), including low levels of resistance to fludioxonil (17). AtrD is an ABC transporter that was linked previously to azole resistance in strains of $B$. cinerea $(15,16)$. 
As part of a region-wide fungicide resistance monitoring program involving $B$. cinerea from blackberry and strawberry, we identified several isolates MR and LR to fludioxonil. The goal of this study was to characterize the molecular basis of resistance to fludioxonil in these isolates. Specific objectives were to investigate (i) potential alterations in genes involved in osmoregulation and promoter rearrangements upstream of $m f_{s} M 2$, (ii) overexpression of $\operatorname{atr} B$ and $\operatorname{atr} D$, (iii) glycerol synthesis and hypersensitivity to salt stress, and (iv) fitness components and cross resistance with dicarboxamide fungicide iprodione in isolates LR and MR to fludioxonil.

\section{MATERIALS AND METHODS}

Isolates and fungicides. $B$. cinerea isolates were obtained from North and South Carolina blackberry fields, a Virginia strawberry field, and Germany (Table 1). Single-spore isolations from symptomatic blackberry and strawberry fruit were conducted as described previously (26). B. cinerea strain Do9_K_A31 (designated Do9 in this study) from a strawberry field in Germany was obtained from Dr. Matthias Hahn, University of Kaiserslautern, Kaiserslautern, Germany and used as MDR1h, group S reference strain (23). Formulated fludioxonil (Scholar SC fungicide, 20.4\% a.i. [vol/vol]; Syngenta, Research Triangle Park, Raleigh, NC) and iprodione (Rovral 4 FL, $41 \%$ a.i. [vol/vol]; Bayer CropScience, Research Triangle Park, NC) were used to determine sensitivities to these fungicides in vitro. Tolnaftate and cycloheximide were purchased from Sigma-Aldrich (St. Louis), dissolved in 100\% ethanol, and diluted in ultrapurified water before being added to the liquid medium. Fungicide stock solutions were adjusted to keep final solvent concentrations $<1.5 \%$ (vol/vol). No significant growth inhibition was observed for strains on media with $<1.5 \%$ ethanol $(20,28)$.

Fungicide sensitivity tests. Categories of fungicide sensitivity to fludioxonil and iprodione were determined using a spore germination assay $(13,36)$. The $\mathrm{EC}_{50}$ values were determined as described previously (20), with minor modifications. Briefly, 5,000 spores were transferred to 0.1-ml 96-microplate cultures. Final concentrations for all compounds were 10, 3, 1, 0.3, 0.1, 0.03 , and $0.01 \mu \mathrm{g} / \mathrm{ml}$. Tests were performed in malt extract broth. After $48 \mathrm{~h}$ of incubation at $22^{\circ} \mathrm{C}$ in darkness, the optical density at wavelength with absorbance at $600 \mathrm{~nm}$ was determined. The experiment was repeated.

DNA extraction, amplification, and sequencing of $\mathrm{mrr} 1$ and the $\boldsymbol{m} \boldsymbol{f} \boldsymbol{s} M 2$ promoter regions. DNA was extracted as described previously (5). The $m r r l$ gene was amplified in two parts; the upstream region was amplified with primers mrr1_atg and TF12_new and the downstream region was amplified with TF1-3_new and TF1-4 (23). The $m f s M 2$ promoter region of $B$. cinerea was amplified with primers Prom_Mfs2_1fw and Prom_Mfs2_2rev (20). Fragments were separated on a $1.5 \%$ agarose gel, stained with ethidium bromide, and visualized under UV-light. The fragment sizes of polymerase chain reaction (PCR) products were verified by comparison with a Low Range Plus DNA Ladder (Fisher Scientific, Waltham, MA). The PCR products were purified using the ExoSAP-IT Purification Kit (USB Corporation, Cleveland, $\mathrm{OH}$ ) following the manufacturer's recommendations. Purified products were sequenced at Clemson University Genomic Center (Clemson, SC). Nucleotide sequences were analyzed, assembled, and aligned with DNASTAR sequence analysis software (DNASTAR, Inc., Madison, WI). B. cinerea strain T4 genome sequences were obtained from GenBank, accession number FQ790286.1.

RNA extraction and gene expression analysis. Isolates were grown on potato dextrose agar (PDA) medium (Difco Laboratories, Sparks, MD) in 9-cm-diameter petri dishes for 10 to 14 days at $22^{\circ} \mathrm{C}$ with 12 -h intervals of fluorescent light and darkness. Conidia were gently scraped off with a sterile, disposable
10- $\mu$ l inoculation loop (VWR International LLC, Radnor, PA) without touching the agar. Then spores were suspended in $2 \mathrm{ml}$ of potato dextrose broth (PDB), with a final spore concentration of 1 to $5 \times 10^{5}$ in $6-\mathrm{cm}$-diameter petri dishes. The spores were incubated for $14.5 \mathrm{~h}$ on a shaker with $100 \mathrm{rpm}$ at $22^{\circ} \mathrm{C}$. The germlings were incubated for another $30 \mathrm{~min}$ in the petri dish either with or without fludioxonil at a final concentration of $1 \mathrm{mg} /$ liter. For RNA isolation, the germlings with the medium were transferred to a 2-ml centrifuge tube and centrifuged for 10,000 rpm for $4 \mathrm{~min}$. The pellet was washed twice with autoclaved, distilled water. Total fungal RNA was isolated using the MasterPure Yeast RNA Purification Kit (EpiCentre Pte. Ltd. Madison, WI) and the RNA quality was checked and quantified by spectrophotometric analysis with a NanoDrop 2000 Spectrophotometer (Thermo Scientific, Waltham, MA). The RNA was then reverse transcribed into cDNA (iScript CDNA Synthesis Kit; BioRad Laboratories, Inc., Hercules, CA). Quantitative reversetranscription PCR was performed as described previously (13), with the exception that we used primers atrBfor/atrBrev and atrDfor/atrDrev for AtrB and AtrD expression analysis, respectively, following published amplification protocols (20). Expression of the genes was calculated according to Pfaffel (31). Transcript levels were normalized against the expression levels of housekeeping genes encoding elongation factor $1 \alpha$ (BC1G_09492.1) and shown as normalized fold-expression levels of noninduced germlings from sensitive (S) strains. Means of at least two biological replicates are shown. Three technical replicates of PCR were used for each biological replicate.

Salt tolerance and determination of glycerol content in germlings. Salt tolerance was investigated on $4 \% \mathrm{NaCl}$-amended minimal medium. A 5-mm-diameter mycelial plug taken from a 3-day-old culture grown on PDA was transferred to the center of the amended medium and dishes were incubated at $22^{\circ} \mathrm{C}$ in the dark. The colony diameter was measured after 3 days of incubation with three replicates per isolate. Mean colony diameter was used to calculate mycelia growth inhibition rate. The experiment was repeated.

The glycerol content was determined in germlings of S, LR, and MR isolates to assess involvement of osmoregulation in the resistance mechanism. Conidia were suspended in $2 \mathrm{ml}$ of PDB in 6-cm-diameter petri dishes with a final concentration of $1 \times 10^{5}$ conidia $/ \mathrm{ml}$. Four dishes per isolate per treatment were incubated for $48 \mathrm{~h}$ on a shaker at $100 \mathrm{rpm}$ and $22^{\circ} \mathrm{C}$. The germlings were incubated for another $1.5 \mathrm{~h}$ in the dishes either with or without fludioxonil at a final concentration of $1 \mathrm{mg} / \mathrm{liter}$. The medium containing the germlings was transferred to a 2-ml impactresistant screw-cap tube (USA Scientific, Inc. Orlando, FL) and centrifuged at 12,000 rpm for $10 \mathrm{~min}$. Medium was removed and the pellet was washed twice with autoclaved distilled water. The volume for each sample was adjusted to $1 \mathrm{ml}$ with water and ground at $4 \mathrm{~m} / \mathrm{s}$ for $40 \mathrm{~s}$ using a FastPrep-24 Tissue and Cell Homogenizer (MP Biomedicals, Solon, OH). Samples were then centrifuged for $10 \mathrm{~min}$ at $12,000 \mathrm{rpm}$ and the glycerol content in the supernatant was determined using an EnzyChrom Glycerol

TABLE 1. Name, origin, and host of isolates used in this study

\begin{tabular}{lllc}
\hline Isolate name & \multicolumn{1}{c}{ Origin } & Host & Year of isolation \\
\hline CA25 & South Carolina & Blackberry & 2010 \\
CB3a & South Carolina & Blackberry & 2011 \\
CO5 & South Carolina & Blackberry & 2010 \\
MC14 & South Carolina & Blackberry & 2010 \\
WM14 & South Carolina & Blackberry & 2010 \\
CO3a & South Carolina & Blackberry & 2011 \\
CB82a & South Carolina & Blackberry & 2011 \\
Wland1 & Virginia & Strawberry & 2012 \\
KC25 & North Carolina & Blackberry & 2011 \\
KC33 & North Carolina & Blackberry & 2011 \\
Do9_K_A31 & Germany & Strawberry & 2009 \\
\hline
\end{tabular}


Assay Kit, following the manufacturer's instructions. After all of the supernatant was removed, the precipitated cell debris was completely dried in an oven for $24 \mathrm{~h}$ at $60^{\circ} \mathrm{C}$. The experiments were repeated two times.

Detection of mutations in the bcsak1, BcOS4, bos5, and BRRG-1 genes. Nucleotide sequences of osmotic regulator genes bcsakl (32), BcOS4 (40), bos5 (39), and BRRG-1 (38) were amplified by PCR from fludioxonil S, LR, and MR isolates. Two overlapping fragments of bcsakl were amplified with primer pairs SAK1F-f (TACCCACTCAACCACCAAC)/SAK1F-r (ATCTTG AATACGGGCGAG) and SAK1B-f (GCTGGTCCTGGAGATA CTAAGA)/SAK1B-r (AAGCACAAGAGAGCACTCCT). These two fragments were assembled into one contiguous sequence covering the entire gene. The core sequence of $\mathrm{BcOS4}$ was amplified with primer pair BOS4C-f (GCTGACGATGAGG AATCA)/BOS4C-r (GTGCTGTAAACACCGACA). The primers were designed based on the $B$. cinerea $\mathrm{T} 4$ genome sequences (1). The bos 5 and $B R R G-1$ genes were amplified with primer pairs Os5-F1/Os5-R1 (39) and Rrg-F1/Rrg-R1, respectively (38).

Assessment of fitness and pathogenicity on fungicidetreated fruit. The following fitness components were investigated for isolates sensitive and resistant to fludioxonil and iprodione. All experiments were repeated.

Mycelial growth on PDA. PDA dishes were inoculated with a 5-mm-diameter mycelial plug taken from 7-day-old culture grown on PDA. The dishes were incubated at $22^{\circ} \mathrm{C}$ in the dark. The colony diameter was measured after 3 days of incubation with three replicates per isolate. Mean colony diameter was calculated for growth rate determination.

Pathogenicity on untreated and fungicide-treated detached fruit. Pathogenicity of each isolate was confirmed by determining its ability to infect and sporulate on strawberry fruit. Commercially grown ripe strawberry fruit were rinsed with sterile water three times for $30 \mathrm{~s}$ each and allowed to air dry. Then, they were placed into plastic boxes (eight strawberry fruit per box for each of the three replicates). When the fruit surface had dried, each fruit was punctured at one point to a depth of $9.5 \mathrm{~mm}$ using a 26G3/8 9.5-mm beveled syringe tip (Becton Dickson \& Co.). The wounds were injected with a $30-\mu l$ droplet of conidia suspension $\left(10^{6}\right.$ spores $\left./ \mathrm{ml}\right)$ prepared in distilled sterile water using the same type of syringe. Most of the conidia suspension formed a droplet on top of the wounded area. After inoculation, the boxes were kept at $22^{\circ} \mathrm{C}$. During the first $24 \mathrm{~h}$, the boxes were sealed with plastic bags to keep the relative humidity high. After 4 days, the lesion diameters were measured.
The ability of isolates resistant to fludioxonil and iprodione to cause disease on fruit treated with fungicides was assessed. Experiments were performed as described above for pathogenicity, tests with minor modifications. Strawberry fruit were sprayed $4 \mathrm{~h}$ prior to inoculation with the recommended label rate of the fungicides Scholar SC (2.5 ml/liter), Switch $62.5 \mathrm{WG}$ (1.9 g/liter; combination of fludioxonil 25\% [vol/vol] and cyprodinil $37.5 \%$ [vol/vol]), and Rovral 4 Flowable ( $2.5 \mathrm{ml} / \mathrm{liter}$ ), using a hand mister to run off. Control fruit were sprayed with sterile distilled water. After 4 days of inoculation, the absence or presence of sporulating lesions was assessed and the lesion diameters were measured and calculated as percentage of the control. The experiment was repeated.

Data analysis. Statistical analysis was performed related to the in vitro and in vivo responses (growth rate, lesion size, disease incidence, control efficacy, and relative expression of atr $B$ and atrD genes) to the experimental factors of experimental replication, isolate, or fungicide treatment. Significant differences were determined through Tukey's honestly significant difference test. All calculations were performed by IBM SPSS Statistics (version 19; IBM SPSS, Armonk, NY) and all tests were performed with $\alpha=0.05$, except as described otherwise.

\section{RESULTS}

Sensitivity of isolates to fludioxonil and iprodione in vitro. In our collection of $412 \mathrm{~B}$. cinerea isolates from blackberry and strawberry, only four isolates were resistant to fludioxonil. Two (Wland1 and CB82a) were categorized as LR based on their ability to germinate with fludioxonil at $1 \mu \mathrm{g} / \mathrm{ml}$ and two (KC25 and $\mathrm{KC} 33$ ) as MR based on their ability to germinate with fludioxonil at $1 \mu \mathrm{g} / \mathrm{ml}$ and show residual growth at $10 \mu \mathrm{g} / \mathrm{ml} . \mathrm{EC}_{50}$ values determined in microtiter assays were 0.16 and $0.26 \mu \mathrm{g} / \mathrm{ml}$ for $\mathrm{LR}$ isolates and 0.32 and $0.38 \mu \mathrm{g} / \mathrm{ml}$ for $\mathrm{MR}$ isolates. Isolates LR or MR but not $\mathrm{S}$ to fludioxonil were $\mathrm{R}$ to tolnaftate and all were $S$ to cycloheximide, consistent with the MDR1 phenotype. Together with two $\mathrm{S}$ isolates, the four LR and MR isolates were also investigated for iprodione sensitivity because phenylpyrroles and dicarboxamides are both believed to target proteins involved in osmotic regulation. All isolates LR and MR to fludioxonil were also $\mathrm{R}$ to iprodione but some fludioxonil-sensitive isolates were LR to iprodione (Table 2).

Expression of multidrug transporter genes atrB and $a t r D$ in isolates with different fludioxonil sensitivity phenotypes. The $\operatorname{atr} B$ genes of MR isolates from blackberry (KC25 and $\mathrm{KC} 33)$ and

TABLE 2. Fitness components for Botrytis cinerea isolates sensitive (S), low-resistant (LR), moderately resistant (MR), or resistant to fludioxonil or iprodione

\begin{tabular}{|c|c|c|c|c|c|c|c|c|c|c|c|c|c|}
\hline \multirow{3}{*}{ Isolates } & & & & & \multicolumn{9}{|c|}{ Fitness components ${ }^{\mathrm{z}}$} \\
\hline & & & & & \multirow{2}{*}{$\frac{3 \text { dai }}{M G(\mathrm{~cm})}$} & \multicolumn{8}{|c|}{4 dai } \\
\hline & \multicolumn{2}{|c|}{ Phenotype $^{\mathrm{x}}$} & \multicolumn{2}{|c|}{$\mathrm{EC}_{50}(\mathrm{mg} / \text { liter })^{\mathrm{y}}$} & & $\mathrm{LS}(\mathrm{cm})$ & SL $(\%)$ & \multicolumn{3}{|c|}{$\mathrm{LS}(\mathrm{cm})$} & \multicolumn{3}{|c|}{ SL $(\%)$} \\
\hline CA25 & $\mathrm{S}$ & $\mathrm{S}$ & $\mathrm{n} / \mathrm{d}$ & $\mathrm{n} / \mathrm{d}$ & $7.3 \mathrm{~b}$ & $3.3 \mathrm{~b}$ & 100.0 & $0.0 \mathrm{a}$ & $\mathrm{n} / \mathrm{d}$ & $0.01 \mathrm{a}$ & 0.0 & $\mathrm{n} / \mathrm{d}$ & $4.2 \mathrm{a}$ \\
\hline CB3a & $\mathrm{S}$ & $S$ & 0.09 & 0.21 & $7.3 \mathrm{ab}$ & $3.3 \mathrm{~b}$ & 100.0 & $0.0 \mathrm{a}$ & 0.0 & $0.01 \mathrm{a}$ & 0.0 & 0.0 & $2.7 \mathrm{a}$ \\
\hline $\mathrm{CO} 5$ & S & LR & $\mathrm{n} / \mathrm{d}$ & $\mathrm{n} / \mathrm{d}$ & $7.6 \mathrm{~b}$ & $3.0 \mathrm{~b}$ & 100.0 & $0.0 \mathrm{a}$ & $\mathrm{n} / \mathrm{d}$ & $2.0 \mathrm{bc}$ & 0.0 & $\mathrm{n} / \mathrm{d}$ & $89.6 \mathrm{~b}$ \\
\hline CB82a & LR & HR & 0.16 & 6.94 & $7.5 \mathrm{~b}$ & $3.2 \mathrm{~b}$ & 100.0 & $1.2 \mathrm{~b}$ & 0.0 & $2.5 \mathrm{~cd}$ & 100.0 & 0.0 & $100.0 \mathrm{~b}$ \\
\hline Wland1 & LR & HR & 0.26 & 3.56 & $7.1 \mathrm{ab}$ & $2.7 \mathrm{a}$ & 100.0 & $1.7 \mathrm{c}$ & $\mathrm{n} / \mathrm{d}$ & $2.2 \mathrm{bcd}$ & 100.0 & $\mathrm{n} / \mathrm{d}$ & $100.0 \mathrm{~b}$ \\
\hline KC25 & MR & HR & 0.32 & $>10$ & $6.1 \mathrm{a}$ & $2.8 \mathrm{a}$ & 100.0 & $1.1 \mathrm{~b}$ & 1.8 & $2.4 \mathrm{bcd}$ & 100.0 & 98.3 & $97.9 \mathrm{~b}$ \\
\hline KC33 & MR & HR & 0.38 & $>10$ & $7.3 \mathrm{~b}$ & $3.3 \mathrm{~b}$ & 100.0 & $1.8 \mathrm{c}$ & 1.5 & $3.2 \mathrm{e}$ & 100.0 & 100.0 & $100.0 \mathrm{~b}$ \\
\hline
\end{tabular}

${ }^{\mathrm{x}}$ Phenotypes were determined based on a spore germination assay using fludioxonil at 0.1 and $10 \mu \mathrm{g} / \mathrm{ml}$ and iprodione at 5 and $50 \mu \mathrm{g} / \mathrm{ml}(36)$. The phenotypic characterization (S, LR, MR, or HR) for some of the isolates listed was reported in an earlier study from our lab (13).

y Fungicide concentration that reduced mycelial growth by $50 \%$.

${ }^{\mathrm{z}}$ Abbreviations: $\mathrm{LS}=$ lesion size (diameter), $\mathrm{SL}=$ sporulating lesions, $\mathrm{MG}=$ mycelial growth, dai $=$ days after inoculation or transfer, and $\mathrm{n} / \mathrm{d}=\mathrm{not}$ determined . Numbers in each column followed by the same letter are not significantly different at $\alpha=0.05$ as determined by analysis of variance. Mean separation was conducted using Tukey's test. 
MDR1h reference isolate Do9 were expressed at $\approx 200$-fold the level of the $\mathrm{S}$ isolates (Fig. 1). Lower expression levels were found for all other phenotypes. The atrB expression levels of LR isolates Wland1 and CB82a were 30- and 100-fold increased, respectively, compared with the $\mathrm{S}$ isolate (Fig. 1). Fludioxonil exposure prior to RNA extraction increased expression of four of the six isolates regardless of their phenotype. They included S isolate CB3a, LR isolate Wland1, and MR isolates KC33 and MDR1h reference isolate Do9.

Analysis of atrD gene expression was conducted because previous reports noted overexpression of this gene in association with MDR phenotypes $(15,16)$. In this study, the expression of atrD was not different among isolates $\mathrm{S}, \mathrm{LR}$, and MR to fludioxonil in untreated or fludioxonil-treated germlings (Fig. 2). Interestingly, the expression of atrD in treated germlings was reduced by $>50 \%$ in all isolates after fludioxonil treatment.

Mutations in the transcription factor $m r r 1$ and the promoter sequences of $m f s M 2$ in strains LR or MR to fludioxonil. Certain mutations in transcription factor Mrr1 influence atrB expression. Therefore, $m r r l$ was sequenced in its entirety from strawberry isolate Wland1 and six blackberry isolates representing $\mathrm{S}$ and $\mathrm{R}$ phenotypes. The $m r r l$ coding regions (including introns) of two $\mathrm{S}$ isolates from blackberry (MC14 and KC20) were 2,444 bp in length and encoded identical amino acid sequences compared with reference isolate $\mathrm{T} 4$, with the exception of two amino-acid variations (L475S and M483V) in isolate MC14 (Fig. 3). S isolate CB3a had an mrrl gene sequence of $2,477 \mathrm{bp}$ in length and revealed several amino-acid variations compared with T4. The mrrl coding region of isolates with resistance phenotypes were 2,444, 2,483, and 2,489 bp in size for isolates Wland1, CB82a, and $\mathrm{KC} 25$ and $\mathrm{KC} 33$ (both 2,489 bp), respectively. Two insertions characteristic for group $\mathrm{S}$ isolates, 18 and $21 \mathrm{bp}$ in length, were discovered in one S, the LR, and both MR isolates from blackberry (Fig. 3). Group $\mathrm{S}$ isolates were considered a special clade of $B$. cinerea and genetically different from the isolates studied (23). In our study, there were only five amino-acid variations among non-group $\mathrm{S}$ isolates but considerably more $(\approx 13$, on average) among group $S$ isolates. The most diversity (up to 38 amino-acid variations) among mrrl gene sequences was found between non-group $\mathrm{S}$ and group $\mathrm{S}$ isolates.

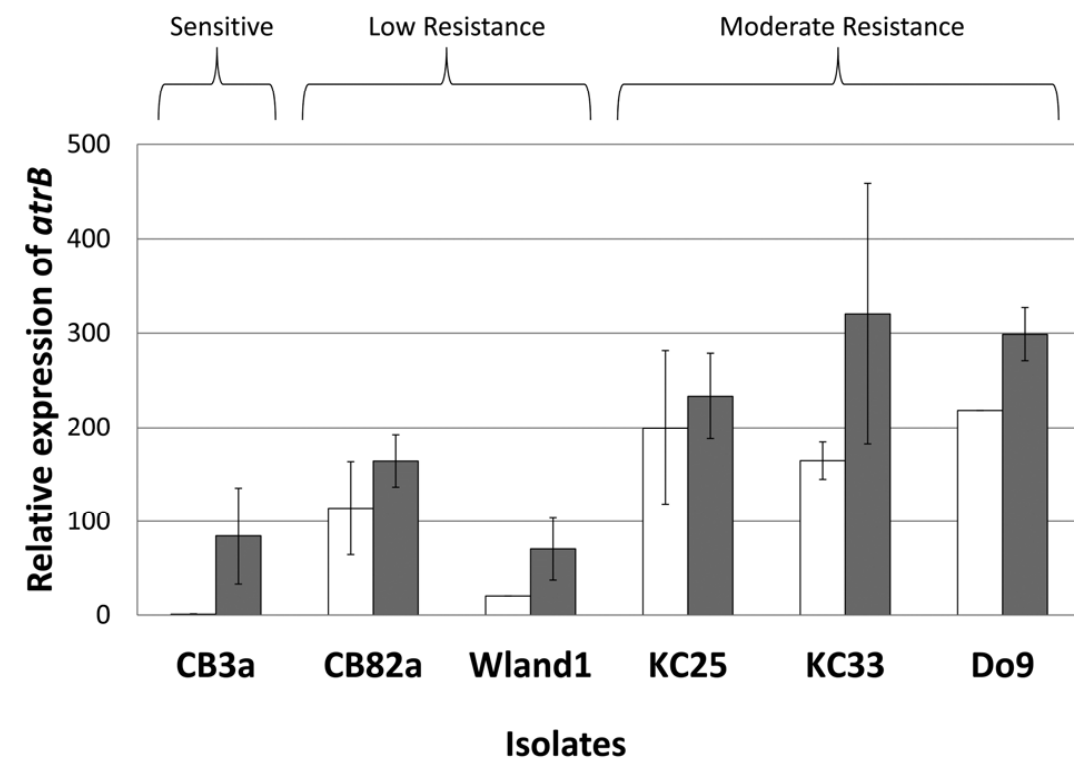

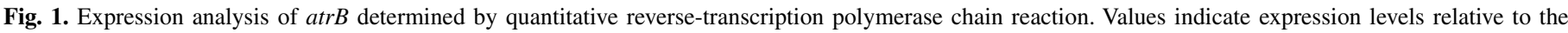
expression of sensitive strain CB3a (which was normalized to 1) with (shaded bars) and without (white bars) fludioxonil treatment prior to RNA extraction.

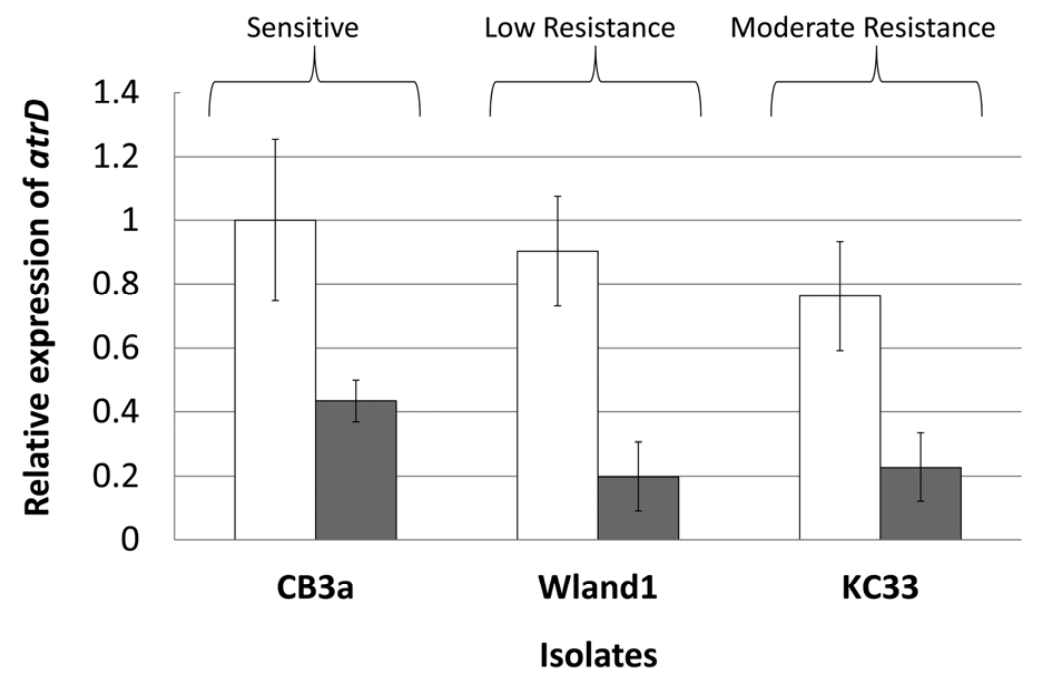

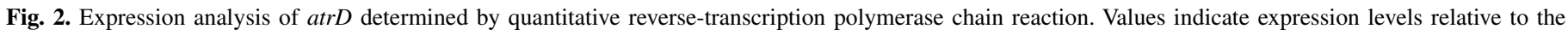
expression of sensitive strain CB3a with (shaded bars) and without (white bars) fludioxonil treatment prior to RNA extraction. 
The MDR1-associated mutation R632I was the only mutation uniquely associated with the fludioxonil MR phenotype (Fig. 3). No point mutations or insertions were detected in the $m f s M 2$ promoter region of isolates LR or MR to fludioxonil, indicating that the isolates did not belong to the MDR2 or MDR3 genotype. The mrrl sequences of isolates CB3a, MC14, KC20, CB82a, $\mathrm{KC} 25$, and $\mathrm{KC} 33$ were submitted to GenBank (accession numbers KJ569562, KJ569563, KJ569564, KJ569565, KJ569566, and KJ569567, respectively).

Evaluation of salt tolerance and glycerol content. Changes in osmoregulatory pathways conferring resistance to phenylpyrroles and dicarboxamides may also influence osmotic sensitivity (11, 17,29,37). Isolates $\mathrm{MR}$ to fludioxonil were significantly more susceptible to salt stress compared with isolates LR and $S$ to fludioxonil (Fig. 4). The sensitivity varied among $\mathrm{S}$ isolates, with a range of 25 to $40 \%$ inhibition. One of each fludioxonil sensitivity phenotype (S, LR, and MR) was subjected to a glycerol content analysis. The three representative isolates roughly produced similar amounts of glycerol in germlings (Fig. 5). Exposure of germlings to fludioxonil prior to extraction increased glycerol content approximately fourfold in all isolates regardless of the phenotype.

Sequencing of genes involved in the osmotic signal transduction pathway. We sequenced large portions of the bcsak1 (1,669 bp), bos5 (1,880 bp), and BRRG-1 (1,655 bp) genes and the core region of the $B c O S 4(1,091 \mathrm{bp})$ gene for the presence of point mutations in $\mathrm{S}, \mathrm{LR}$, and MR isolates. No point mutations were identified that were uniquely associated with the fludioxonil
LR or MR phenotype (data not shown). Both MR isolates had a 5-bp deletion in an intron at nucleotide position 1,261 of the bcsakl gene (data not shown); the sequences from one $\mathrm{S}$ and one MR isolate were submitted to GenBank (accession numbers KF964016 [CB3a] and KF964017 [KC25]). Analysis of 45 fludioxonil-sensitive isolates that originated from the same site as the MR isolates (location $\mathrm{KC}$ ), and 11, 16, 4, and 24 isolates from locations $\mathrm{MC}, \mathrm{CO}, \mathrm{CA}$, and $\mathrm{WM}$, respectively, revealed that the deletion was unique to the MR isolates (data not shown). However, the deletion was absent in the reference strain from Germany MR to fludioxonil, suggesting that the deletion is not linked to the MDR1 phenotype.

Fitness components of isolates $\mathrm{S}$, LR, and MR to fludioxonil. Cross resistance between phenylpyrroles and dicarboxamides is common because products of both fungicide classes are believed to be involved in the inhibition of proteins involved in osmotic signal transduction. All isolates LR and MR to fludioxonil were also $\mathrm{R}$ to iprodione. The fludioxonil-sensitive isolates however, revealed various iprodione sensitivity phenotypes (Table 2 ). There were no significant differences in mycelial growth rates and lesion size between $\mathrm{S}$ isolates and LR and MR isolates, with one exception. Mycelium growth was slower for isolate $\mathrm{KC} 25$ compared with most isolates, including four $\mathrm{S}$ and two dual-R isolates (Table 2). The same isolate also produced a significantly smaller lesion $(P<0.05)$ size on inoculated fruit compared with all other isolates. The other MR isolate, $\mathrm{KC} 33$, did not suffer a fitness penalty in our study. All $\mathrm{S}$ and $\mathrm{R}$ isolates were able to produce sporulating lesions on detached fruit (Table 2).

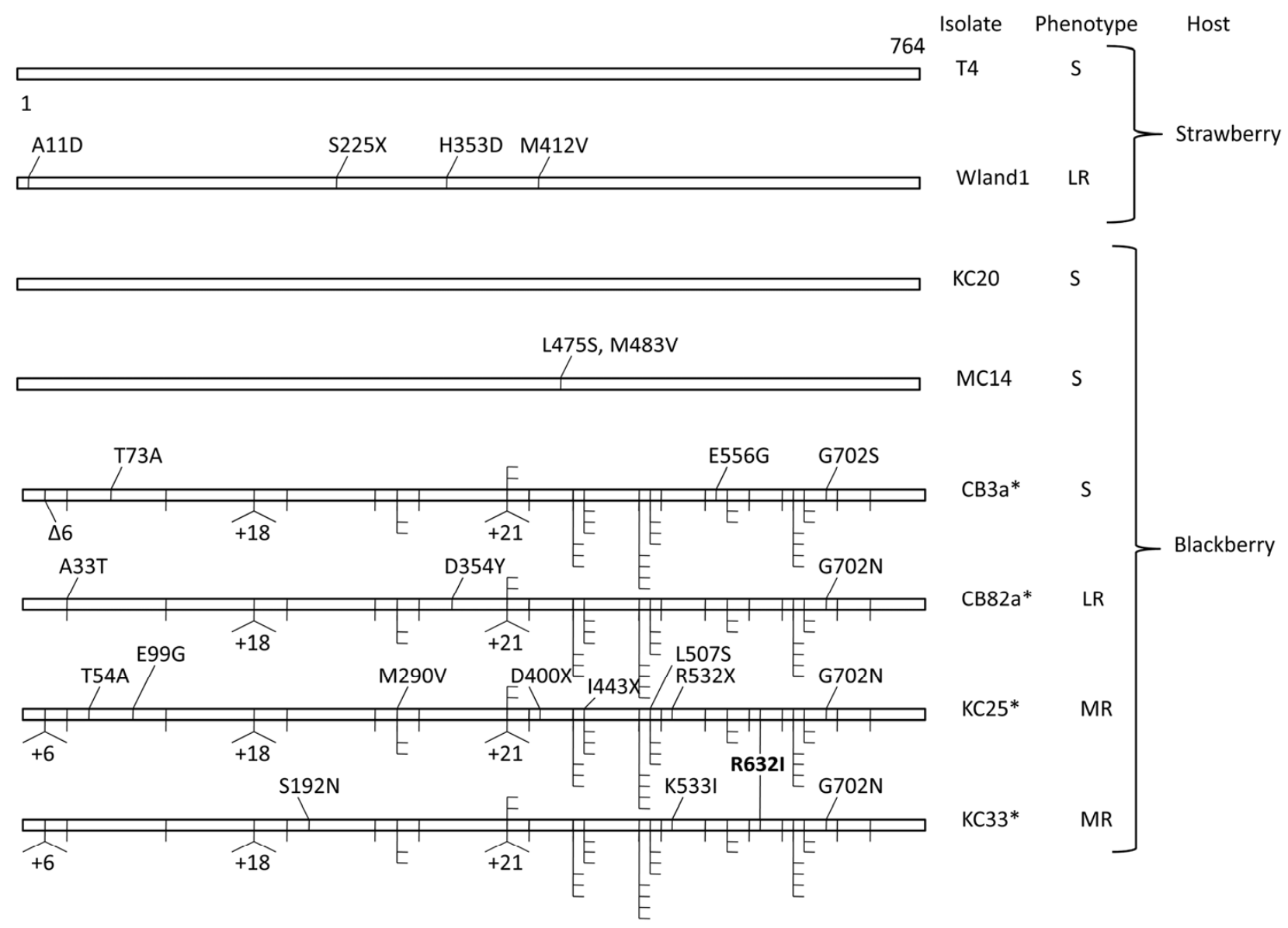

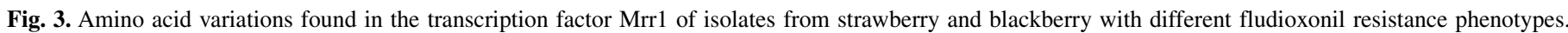

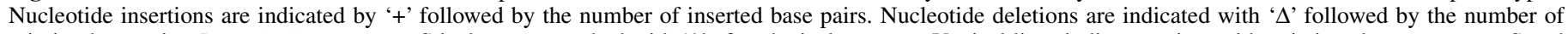

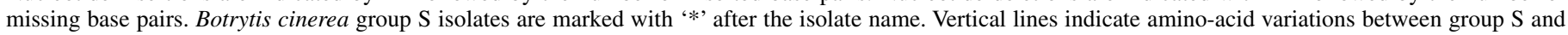

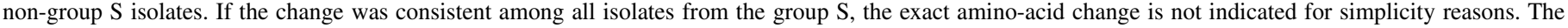

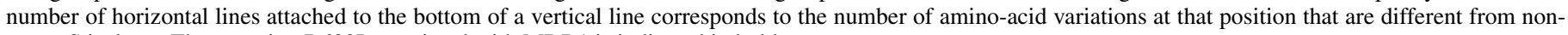
group S isolates. The mutation R632I associated with MDR1 is indicated in bold. 
The ability of isolates S, LR, and MR to fludioxonil to cause disease on fruit sprayed with fungicides was assessed to determine whether these isolates can withstand label rates of fungicide. Isolates $\mathrm{S}$ to fludioxonil were completely controlled with Scholar SC fungicide, but isolates LR and MR to fludioxonil developed disease with sporulating lesions. Both isolates MR to fludioxonil were also able to develop sporulating lesions on fruit treated with Switch $62.5 \mathrm{WG}$, a commercially available combination product of fludioxonil and AP fungicide cyprodinil, indicating that the AP fungicide component in this mixture did not prevent disease formation. This is consistent with the in vitro resistance to cyprodinil and fludioxonil and the MDR1 phenotype. Isolates R to iprodione in vitro, including the isolates $\mathrm{R}$ to both iprodione and fludioxonil, developed disease with sporulating lesions on fruit treated with label rates of Rovral 4 Flowable. The isolates S to iprodione were almost completely controlled in the Rovral 4 Flowable treatment (Table 2).

\section{DISCUSSION}

Although resistance in $B$. cinerea to many classes of fungicides, including benzimidazoles, quinone outside inhibitors, succinate dehydrogenase inhibitors, hydroxyanalides, anilinopyrimidines, and dicarboxamides, is fairly common $(3,21-23,27,35)$, resistance to phenylpyrroles, including fludioxonil, is still rare. In this study, we show that resistance to fludioxonil is emerging in small fruit crops in the eastern United States. Resistance to fludioxonil is likely to increase in light of resistance problems with many other compounds of different modes of action. The low frequencies of isolates LR and MR to fludioxonil in our collection of 412 isolates from blackberry and strawberry is consistent with other studies that found that fludioxonil resistance is either absent or rare in southeastern strawberry fields $(6,7)$ and that it is likely not of practical relevance for fruit growers at this time. The low frequency of LR and MR isolates validates the idea that phenylpyrroles are still valuable tools for rotations or mixtures with other site-specific fungicides for effective gray mold control. However, the here-documented emergence of such strains in United States berry crop production fields should be considered in disease management strategies meant to delay selection. Examples already exist where large portions of field isolates gained partial resistance to fludioxonil due to fungicide selection (23).

In this study, the $\mathrm{EC}_{50}$ values for fludioxonil in $\mathrm{MR}$ isolates were comparable with those found in isolates from strawberry

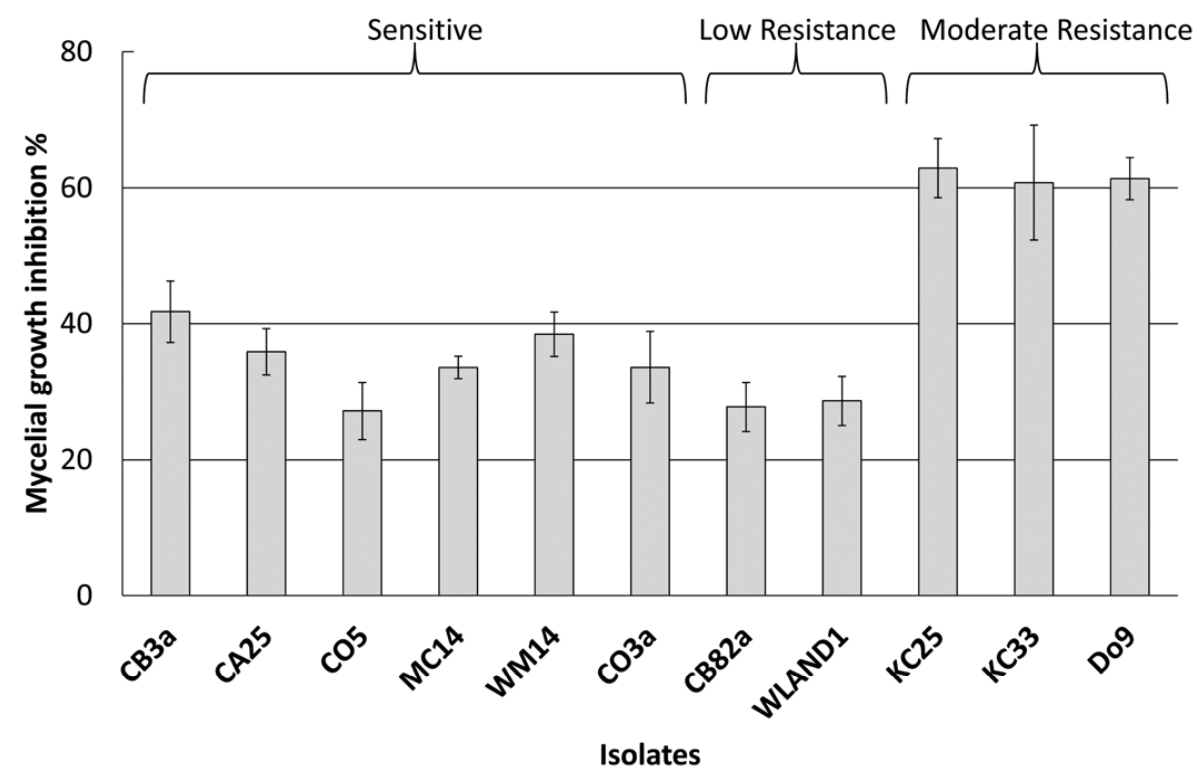

Fig. 4. Mycelial growth inhibition of isolates sensitive, low-resistant, and moderately resistant to fludioxonil on minimal medium amended with $4 \% \mathrm{NaCl}$.

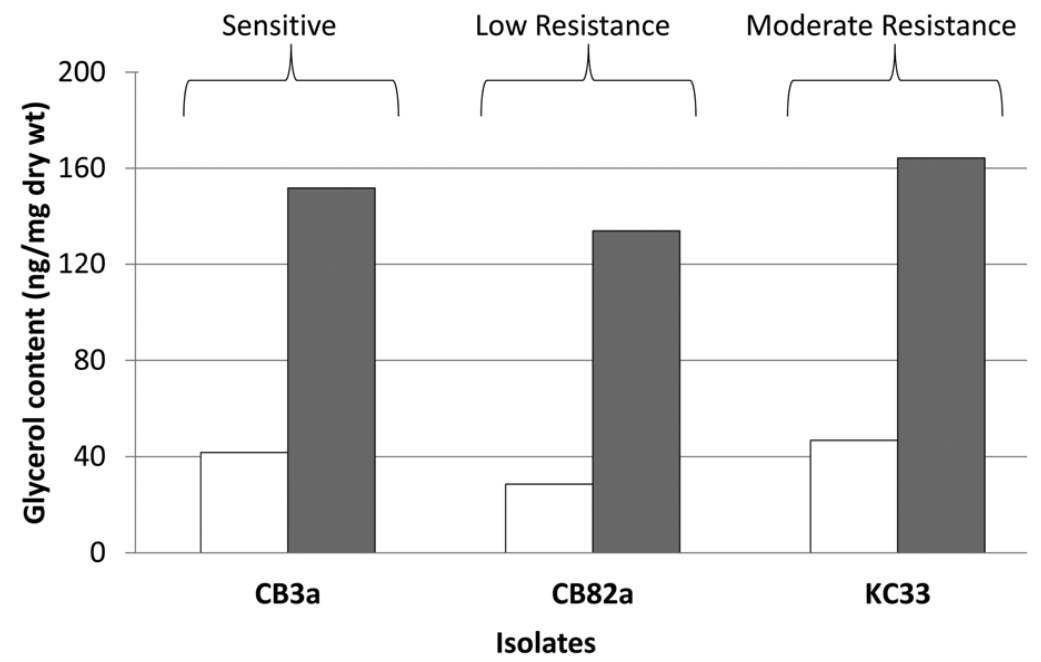

Fig. 5. Glycerol content in mycelia of Botrytis cinerea isolates sensitive, low-resistant, and moderately resistant to fludioxonil. Mycelia subjected to fludioxonil treatment prior to analysis are indicated in shaded bars. 
$(20,23)$ and grapevines $(20,22,28)$ in Europe. Moderate resistance to fludioxonil in $B$. cinerea isolates from European grape was designated MDR1 and conferred by mutation R632I in transcription factor Mrr1, leading to overexpression of ABC transporter gene $\operatorname{atr} B(20)$. Both MR isolates analyzed in this study contained the R632I mutation in Mrr1 associated with MDR1 but atrB overexpression levels were similar to MDR1h reference isolate Do9 (20,33). MDR1h isolates revealed higher levels of resistance to cyprodinil and fludioxonil with generally higher overexpression of $a t r B$ compared with MDR1 (23). Our isolates, however, did not possess the MDR1h's signature L497 deletion in Mrr1 (23). This suggests that this deletion may not exclusively be responsible for the high levels of $\operatorname{atr} B$ overexpression. MR isolates were $\mathrm{R}$ to tolnaftate but $\mathrm{S}$ to cyclohexamide, further confirming the MDR1 phenotype (20). The existence of MDR1 in the United States suggests that this resistance mechanism is important for the adaptation of $B$. cinerea to fludioxonil pressure. Because of the great distance between the two continents, the Atlantic Ocean separating the two land masses, and the significant genetic variation in the $m r r l$ gene between the German MDR1h reference strain and the isolates from the United States (data not shown), it is likely that the same resistance mechanism developed independently in the two continents.

The LR phenotype may be caused by overexpression of atrB as well, albeit at a lower expression level (up to 100-fold in this study). However, variations associated with the MDR1 or MDR1h phenotypes were not found in the mrrl genes of LR isolates. An investigation into genes involved in osmotic regulation, including bcsakl, bos5, BcOS4, and BRRG-1, revealed no nucleotide variations associated with resistance between $S$, LR, or MR isolates. Furthermore, we found no evidence in MR or LR isolates that would suggest involvement of the MDR2 genotype, which is characterized by promoter region rearrangements upstream of the $m f s M 2$ promoter region and reduced sensitivity to cycloheximide (data not shown) (20).

The lack of fitness cost of isolate KC33, MR to fludioxonil, in this study indicates that overexpression of $a t r B$ may not necessarily come with a fitness cost. However, MR isolate KC25 did have slower growth rates on fruit, which is consistent with reports for a fludioxonil-resistant Penicillium expansum field isolate (25). Laboratory mutants of $B$. cinerea revealed two types of fludioxonil-resistant phenotype; one R to osmotic stress (FLDosm/r) and another $\mathrm{S}$ to high osmotic stress (FLDosm/s). Only FLDosm/s had fitness cost (44) that was later confirmed in field studies (43). In this study, both MR isolates were hypersensitive to salt in the form of exposure to $4 \% \mathrm{NaCl}$ despite having different fitness phenotypes. Neither salt-stress hypersensitivity nor a linkage to iprodione resistance has been reported for MDR1 and MDR1h strains before, and are not necessarily expected because MDR phenotypes are purely based on efflux transporter overexpression. Whether hypersensitivity to salt stress and MR to fludioxonil are linked is unknown due to the low sample size investigated in this study. If they are linked, it could be exploited for management purposes. The increased sensitivity to salt stress was not associated with mutations in the bcsakl, bos5, BRRG-1, and BcOS4 genes involved in the osmoregulation pathway (data not shown). It is possible that their expression pattern or other genes involved in osmoregulation are responsible for this phenotype. Increased sensitivity to salt stress was also found in Botrytis isolates from apple orchards in Washington State (43). Numerous studies reported the linkage of the fludioxonil-resistant phenotype and hypersensitivity to salt stress in laboratory mutants with dysfunctional genes involved in osmoregulation in Neurospora crassa $(10,11,29)$ as well as in $B$. cinerea $(9,32,38-40)$. Previous studies on the mode of actions of phenylpyrrole fungicides in $N$. crassa indicated that this class of fungicides affects the osmotic signal transduction pathway (10) and our results indicate that this mode of action is also true for B. cinerea.
All isolates, regardless of fludioxonil resistance phenotype, accumulated glycerol when exposed to fludioxonil, which increases the internal turgor pressure. In other fungi, the exposure to fludioxonil increased the intracellular glycerol content for both $\mathrm{S}$ and MR isolates but not highly $\mathrm{R}$ isolates in $P$. digitatum (18). The authors concluded that the mode of action of fludioxonil in $P$. digitatum is probably the MAPK pathway that stimulates glycerol synthesis in $\mathrm{S}$ and MR. We saw the same phenomenon in our $\mathrm{S}$, $\mathrm{LR}$, and MR isolates, which confirms the involvement of fludioxonil in the MAPK pathway in B. cinerea but also suggests that the mechanism conferring LR and MR may not be directly associated with the regulation of glycerol synthesis.

We focused our mrrl gene analysis on characterization of fludioxonil phenotypes and therefore sequenced three S, two LR (one from blackberry and one from strawberry), and two MR isolates. Among those strains, one S, one LR (blackberry), and two MR contained the $B$. cinerea group $\mathrm{S}$ signature consistent with two insertions (18 and $21 \mathrm{bp}$ ) in $\mathrm{mrrl}$ (Fig. 3), which were first identified in isolates from German strawberry fields (23). Thus far, group $\mathrm{S}$ isolates from Germany have been exclusively found in strawberry fields and are believed to be a result of differential host adaptation or reduced genetic exchange (23). The presence of group $\mathrm{S}$ and non-group $\mathrm{S}$ strains in populations from blackberry in this study would indicate that group $S$ strains are not a result of host adaptation. Group $S$ strains might be sexually isolated from other clades as suggested above, given the large numbers of amino-acid variation in group $\mathrm{S}$ compared with the non-group $\mathrm{S}$ strains. However, many more isolates and different genes would need to be sequenced to validate this hypothesis.

The LR and MR phenotypes were capable of developing disease on fruit sprayed with label rates of Switch $62.5 \mathrm{WG}$, Scholar SC, or Rovral 4 Flowable. Together with our fitness data, this indicates that these resistance phenotypes are likely being selected for by these fungicides in the field and that they may be able to compete with $\mathrm{S}$ isolates in the absence of selection pressure. This finding is consistent with a study from Germany that showed establishment of MDR-like phenotypes in both vineyards and strawberry fields after application of different spray programs containing Signum (pyraclostrobin and boscalid), Teldor (fenhexamid), and Switch 62.5WG (23).

In conclusion, $B$. cinerea strains with resistance to fludioxonil are emerging in strawberry and blackberry fields of the southern United States. The mechanism of resistance in MR isolates is the same as described in European isolates, emphasizing the importance and relevance of this mechanism of resistance for fludioxonil. However, the low number of isolates examined does not allow firm conclusions regarding to what extent this mechanism of resistance is present in MR isolates. The role of atrB overexpression in LR isolates is still unclear.

\section{ACKNOWLEDGMENTS}

Technical contribution number 6127 of the Clemson University Experiment Station. This material is based upon work supported by the CSREES/USDA, under project number SC-1000642 and USDA-NIFA project no. 2010-51181-21113. Dr. D. Fernández-Ortuño has received funding from the Marie Curie COFUND program "U-Mobility" cofinanced by Universidad de Málaga and the European Community's Seventh Framework Program under Grant Agreement Number 246550. We thank P. K. Bryson (Clemson University) for technical support and S. Scott (Clemson University) for letting us use some of his equipment. We thank Dr. Matthias Hahn, University of Kaiserslautern, for sending us $B$. cinerea isolates and for assistance in $\mathrm{mrrl}$ gene sequence analysis.

\section{LITERATURE CITED}

1. Amselem, J., Cuomo, C. A., van Kan, J. A. L., Viaud, M., Benito, E. P., Couloux, A., Coutinho, P. M., de Vries, R. P., Dyer, P. S., Fillinger, S., 
Fournier, E., Gout, L., Hahn, M., Kohn, L., Lapalu, N., Plummer, K. M., Pradier, J.-M., Eveline, Q., Sharon, A., Simon, A., ten Have, A., Tudzynski, B., Tudzynski, P., Wincker, P., Andrew, M., Veronique, A., Beever, R. E., Beffa, R., Benoit, I., Bouzid, O., Brault, B., Chen, Z., Choquer, M., Jerome, C., Cotton, P., Danchin, E. G., Da Silva, C., Angelique, G., Giraud, C., Giraud, T., Gonzalez, C., Grossetete, S., Ulrish, G., Henrissat, B., Howlett, B. J., Kodira, C., Kretschmer, M., Lappartient, A., Leroch, M., Levis, C., Mauceli, E., Cecile, N., Oeser, B., Pearson, M., Poulain, J., Poussereau, N., Quesneville, H., Rascle, C., Schumacher, J., Beatrice, S., Sexton, A., Silva, E., Sirven, C., Soanes, D. M., Talbot, N. J., Templeton, M., Yandava, C., Yarden, O., Zeng, Q., Rollins, J. A., Lebrun, M.-H., and Dickman, M. 2011. Genomic analysis of the necrotrophic fungal pathogens Sclerotinia sclerotiorum and Botrytis cinerea. PLoS Genet. 7(8):e1002230. Online publication. doi:10.1371/ journal.pgen. 1002230

2. Bahn, Y. S., Kojima, K., Cox, G. M., and Heitman, J. 2006. A unique fungal two-component system regulates stress responses, drug sensitivity, sexual development, and virulence of Cryptococcus neoformans. Mol. Biol. Cell 17:3122-3135.

3. Bardas, G. A., Veloukas, T., Koutita, O., and Karaoglanidis, G. S. 2010. Multiple resistance of Botrytis cinerea from kiwifruit to SDHIs, QoIs and fungicides of other chemical groups. Pest Manage. Sci. 66:967-973.

4. Brent, K. J., and Hollomon, D. W. 2007. Pages 16 in: Fungicide Resistance: The Assessment of Risk. FRAC Monogr. No. 2. Global Crop Protection Federation, Brussels.

5. Chi, M. H., Park, S. Y., and Lee, Y. H. 2009. A quick and safe method for fungal DNA extraction. Plant Pathol. J. 25:108-111.

6. Fernández-Ortuño, D., Bryson, P. K., Grabke, A., and Schnabel, G. 2013. First report of fludioxonil resistance in Botrytis cinerea from a strawberry field in Virginia. Plant Dis. 97:848.

7. Fernández-Ortuño, D., Chen, F., and Schnabel, G. 2013. Distribution and characterization of resistance to cyprodinil and lack of fludioxonil resistance in Botrytis cinerea isolates from strawberry in North and South Carolina. Plant Dis. 97:81-85.

8. Fernández-Ortuño, D., Grabke, A., Bryson, P. K., Rouse, R. J., Rollins, P., and Schnabel, G. First report of fludioxonil resistance in Botrytis cinerea, the causal agent of gray mold disease, from strawberry fields in Maryland and South Carolina. Plant Dis. (In press.)

9. Fillinger, S., Ajouz, S., Nicot, P. C., Leroux, P., and Bardin, M. 2012. Functional and structural comparison of pyrrolnitrin- and iprodioneinduced modifications in the class III histidine-kinase Bos1 of Botrytis cinerea. PLoS One 7:e42520. Online publication. doi:10.1371/journal. pone. 0042520

10. Fujimura, M., Ochiai, N., Ichiishi, A., Usami, R., Horikoshi, K., and Yamaguchi, I. 2000. Sensitivity to phenylpyrrole fungicides and abnormal glycerol accumulation in $O s$ and $C u t$ mutant strains of Neurospora crassa. J. Pestic. Sci. 25:31-36.

11. Fujimura, M., Ochiai, N., Oshima, M., Motoyama, T., Ishiishi, A., Usami, R., Horikoshi, K., and Yamaguchi, I. 2003. Putative homologs of SSK22 MAPKK kinase and PBS2 MAPK kinase of Saccharomyces cerevisiae encoded by $o s-4$ and $o s-5$ genes for osmotic sensitivity and fungicide resistance in Neurospora crassa. Biosci. Biotechnol. Biochem. 67:186191.

12. Gehmann, K., Nyfeler, R., Leadbeater, A. J., Nevill, D., and Sozzi, D. 1990. CGA:173506: a new phenylpyrrole fungicide for broad-spectrum disease control. Proc. Br. Crop Prot. Conf. Pests Dis. 2:369-376.

13. Grabke, A., Fernández-Ortuño, D., Amiri, A., Li, X., Peres, N. A., Smith, P., and Schnabel, G. 2014. Characterization of iprodione resistance in Botrytis cinerea from strawberry and blackberry. Phytopathology 104: 396-402.

14. Hagiwara, D., Matsubayashi, Y., Marui, J., Frukawa, K., Yamashino, T., Kanamaru, K., Kato, M., Abe, K., Kobayashi, T., and Mizuno, T. 2007. Characterization of the NikA histidine kinase implicated in the phosphorelay signal transduction of Aspergillus nidulans, with special reference to fungicide responses. Biosci. Biotechnol. Biochem. 71:844847.

15. Hayashi, K., Schoonbeek, H., and de Waard, M. A. 2002. Expression of the $\mathrm{ABC}$ transporter BcatrD from Botrytis cinerea reduces sensitivity to sterol demethylation inhibitor fungicides. Pestic. Biochem. Physiol. 73:110-121.

16. Hayashi, K., Schoonbeek, H., Sugiura, H., and de Waard, M. A. 2001. Multidrug resistance in Botrytis cinerea associated with decreased accumulation of the azole fungicide oxpoconazole and increased transcription of the ABC transporter gene BcatrD. Pestic. Biochem. Physiol. 70:168-179.

17. Jones, C. A., Greer-Phillips, S. E., and Borkovich, K. A. 2007. The response regulator RRG-1 functions upstream of a mitogen-activated protein kinase pathway impacting asexual development, female fertility, osmotic stress, and fungicide resistance in Neurospora crassa. Mol. Biol. Cell 18:2123-2136.
18. Kanetis, L., Forster, H., Jones, C. A., Borkovich, K. A., and Adaskaveg, J. E. 2008. Characterization of genetic and biochemical mechanisms of fludioxonil and pyrimethanil resistance in field isolates of Penicillium digitatum. Phytopathology 98:205-214.

19. Kojima, K., Takano, Y., Yoshimi, A., Tanaka, C., Kikuchi, T., and Okuno, T. 2004. Fungicide activity through activation of a fungal signalling pathway. Mol. Microbiol. 53:1785-1796.

20. Kretschmer, M., Leroch, M., Mosbach, A., Walker, A.-S., Fillinger, S., Mernke, D., Schoonbeek, H.-J., Pradier, J.-M., Leroux, P., de Waard, M. A., and Hahn, M. 2009. Fungicide-driven evolution and molecular basis of multidrug resistance in the field populations of the grey mould fungus Botrytis cinerea. PLoS Pathog. 5:e1000696.

21. Latorre, B. A., and Torres, R. 2012. Prevalence of isolates of Botrytis cinerea resistant to multiple fungicides in Chilean vineyards. Crop Prot. 40:49-52.

22. Leroch, M., Kretschmer, M., and Hahn, M. 2011. Fungicide resistance phenotypes of Botrytis cinerea isolates from commercial vineyards in South West Germany. J. Phytopathol. 159:63-65.

23. Leroch, M., Plesken, C., Weber, R. W. S., Kauff, F., Scalliet, G., and Hahn, M. 2013. Gray mold populations in German strawberry fields show multiple fungicide resistance and are dominated by a novel clade close to Botrytis cinerea. Appl. Environ. Microbiol. 79:159-167.

24. Leroux, P. 1996. Recent developments in the mode of action of fungicides. Pestic. Sci. 47:191-197.

25. Li, H. X., and Xiao, C. L. 2008. Characterization of fludioxonil-resistant and pyrimethanil-resistant phenotypes of Penicillium expansum from apple. Phytopathology 98:427-435.

26. Li, X. P., Fernández-Ortuño, D., Chai, W., Wang, F., and Schnabel, G. 2012. Identification and prevalence of Botrytis spp. from blackberry and strawberry fields of the Carolinas. Plant Dis. 96:1634-1637.

27. Li, X. P., Fernández-Ortuño, D., Chen, S., Grabke, A., Luo, C. X., Bridges, W. C., and Schnabel, G. Location-specific fungicide resistance profiles and evidence for stepwise accumulation of resistance in Botrytis cinerea. Plant Dis. (In press.)

28. Mernke, D., Dahm, S., Walker, A.-S., Lalève, A., Fillinger, S., Leroch, M., and Hahn, M. 2011. Two promoter rearrangements in a drug efflux transporter gene are responsible for the appearance and spread of multidrug resistance phenotype MDR2 in Botrytis cinerea isolates in French and German vineyards. Phytopathology 101:1176-1183.

29. Noguchi, R., Banno, S., Ichikawa, R., Fukumori, F., Ichiishi, A., Kimura, M., Yamaguchi, I., and Fujimura, M. 2007. Identification of OS-2 MAP kinase-dependent genes induced in response to osmotic stress, antifungal agent fludioxonil, and heat shock in Neurospora crassa. Fungal Genet. Biol. 44:208-218.

30. Ochiai, N., Fujimura, M., Oshima, M., Motoyama, T., Ichiishi, A., Yamada-Okabe, H., and Yamaguchi, I. 2002. Effects of iprodione and fludioxonil on glycerol synthesis and hyphal development in Candida albicans. Biosci. Biotechnol. Biochem. 66:2209-2215.

31. Pfaffl, M. W. 2001. A new mathematical model for relative quantification in real-time RT-PCR. Nucleic Acids Res. 29:45-50.

32. Segmuller, N., Ellendorf, U., Tudzynski, B., and Tudzynski, P. 2007. BcSAK1, a stress-activated mitogen-activated protein kinase, is involved in vegetative differentiation and pathogenicity in Botrytis cinerea. Eukaryot. Cell 6:211-221.

33. Vermeulen, T., Schoonbeek, H., and de Waard, M. A. 2001. The ABC transporter BcatrB from Botrytis cinerea is a determinant of the activity of the phenylpyrrole fungicide fludioxonil. Pest Manage. Sci. 57:393-402.

34. Vigntelli, A., Hilber-Bodmer, M., and Hilber, U. W. 2002. Genetic analysis of resistance to the phenylpyrrole fludioxonil and the dicarboximide vinclozolin in Botryotinia fuckeliana (Botrytis cinerea). Mycol. Res. 106:329-335.

35. Weber, R. W. S. 2011. Resistance of Botrytis cinerea to multiple fungicides in northern German small-fruit production. Plant Dis. 95:12631269.

36. Weber, R. W. S., and Hahn, M. 2011. A rapid and simple method for determining fungicide resistance in Botrytis. J. Plant Dis. Prot. 118:17-25.

37. Yamashita, K., Shiozawa, A., Watanabe, S., Fukumori, F., Kimura, M., and Fujimura, M. 2008. ATF-1 transcription factor regulates the expression of ccg-1 and cat-1 genes in response to fludioxonil under OS-2 MAP kinase in Neurospora crassa. Fungal Genet. Biol. 45:1562-1569.

38. Yan, L., Yang, Q., Jiang, J., Michailides, T., and Ma, Z. 2011. Involvement of a putative response regulator Brrg-1 in the regulation of sporulation, sensitivity to fungicides, and osmotic stress in Botrytis cinerea. Appl. Environ. Microbiol. 90:215-226.

39. Yan, L., Yang, Q., Sundin, G. W., Li, H., and Ma, Z. 2010. The mitogenactivated protein kinase kinase BOS5 is involved in regulating vegetative differentiation and virulence in Botrytis cinerea. Fungal Genet. Biol. 47:753-760.

40. Yang, Q., Yan, L., Gu, Q., and Ma, Z. 2012. The mitogen-activated 
protein kinase kinase kinase $\mathrm{BcOs} 4$ is required for vegetative differentiation and pathogenicity in Botrytis cinerea. Appl. Environ. Microbiol. 96:481-492.

41. Yoshimi, A., Kojima, Y., Takano, Y., and Tanaka, C. 2005. Group III histidine kinase is a positive regulator of Hog1-type mitogen-activated protein kinase in filamentous fungi. Eukaryot. Cell 4:1820-1828.

42. Zhang, Y., Lamm, R., Pillonel, C., Lam, S., and Xu, J. R. 2002. Osmoregulation and fungicide resistance: the Neurospora crassa os-2 gene encodes a HOG1 mitogen-activated protein kinase homologue. Appl.
Environ. Microbiol. 68:532-538.

43. Zhao, H., Kim, Y. K., Huang, L., and Xiao, C. L. 2010. Resistance to thiabendazole and baseline sensitivity to fludioxonil and pyrimethanil in Botrytis cinerea populations from apple and pear in Washington State. Postharvest Biol. Technol. 56:12-18.

44. Ziogas, B. N., Markoglou, A. N., and Spyropoulou, V. 2005. Effect of phenylpyrrole-resistance mutations on ecological fitness of Botrytis cinerea and their genetical basis in Ustilago maydis. Eur. J. Plant Pathol. 113:83-100. 